\title{
Adaptive Collaborative Web-Based Courses
}

\author{
Rosa M. Carro ${ }^{1,2}$, Alvaro Ortigosa ${ }^{1,2}$, and Johann Schlichter ${ }^{1}$ \\ ${ }^{1}$ Institute for Informatics, Technical University of Munich \\ 85748 Munich, Germany \\ schlichter@in.tum.de \\ ${ }^{2}$ Escuela Politecnica Superior, Universidad Autonoma de Madrid \\ 28049 Madrid, Spain \\ \{rosa.carro, alvaro.ortigosa\}@ii.uam.es
}

\begin{abstract}
In this paper we present the way adaptation techniques and collaboration capabilities have been seamlessly integrated to dynamically generate adaptive collaborative Web-based courses. In these courses collaborative and noncollaborative tasks are dynamically proposed. The most appropriate tasks to be performed, the time at which they are available, the type of collaboration tools provided and the most suitable partners for cooperating with, among others, are decided for each user by considering her personal features, knowledge and behavior while interacting with the course. The student actions while learning individually are considered when deciding the collaboration aspects. Student actions during the collaboration can affect the course evolution. A system to support the generation and delivering of this type of courses is being implemented.
\end{abstract}

\section{Motivation and Goals}

Adaptive hypermedia has proved to be effective for the development of adaptive Web-based courses. In these courses students are guided during the learning process. Each course is adapted by selecting the most suitable topics to be presented at any particular moment, their structure and organization, the navigational options available at each step, the help offered and the multimedia contents for page generation, taking into account the student features, preferences and actions [1]. The main goal is for every student to learn in the most efficient way. Each student can interact with an adaptive course, usually alone, in her own time frame, and learn at her own pace.

In addition, the use of collaboration tools in distance learning allows the users to communicate among each other, to share their knowledge, to work together on the resolution of problems and to discuss topics or doubts, among others. It reduces student isolation and facilitates the development of reasoning skills such as making ideas explicit, arguing, interacting with others to build a common solution, etc. [2].

Remarkable works concerning adaptive teaching can be found in the literature (see [1] for a survey on existing systems and tools). Interesting works on collaborative learning [3] have also been developed, some of them focusing on the interaction protocols [2] or on the support of teacher-student cooperation [4].

1 This work is being partially supported by the German Academic Exchange Service (DAAD) and the Spanish CICYT, projects TIC2001-0685-C02-01 and TIC2002-01948. 
Our approach deals with the integration of collaboration capabilities in Web-based adaptive courses and with the use of adaptation methods and techniques for the personalization of not only the course contents but also of the collaboration aspects. It consists not just of putting adaptive courses and collaborative tools together, but of integrating adaptation and collaboration in a seamless way. Therefore, the users can benefit not only from both of them separately, but also from a combination of the two.

With this purpose, we are extending the TANGOW system [6], which has been used during the last years for dynamic generation of adaptive Web-based courses, to create a system for the generation of collaborative adaptive Web-based courses. In this new system, the progress of the student while learning topics, solving exercises individually, navigating through the course, etc. is taken into consideration for decisions about collaborative aspects such as the presence or absence of collaborative tasks or the group formation, among others. In a similar way, the student's actions while performing collaborative tasks can affect the remaining course evolution.

\section{Adaptive Collaborative Courses}

While interacting with an adaptive collaborative course the students will be: i) guided individually through the course contents, ii) invited to cooperate with other students in the resolution of certain problems at specific points of the course and iii) able to share ideas, contact other students, and ask/answer questions about the course. Concerning the cooperative activities, aspects such as the particular collaborative exercises proposed, the time at which they are presented, the collaboration tools available, the type of collaboration, and the partners who a student will interact with, among others, are decided by taking into account each user's personal features, knowledge and behavior while taking the course. Some of these adaptation capabilities are explained next.

Collaborative Tasks Proposed. The time at which certain collaborative tasks are presented can differ depending on the user taking the course. Besides, collaboration can be available after the student has performed certain tasks or at any time. Sometimes it can be convenient to present different collaborative tasks, related or not to the same subject, to different students. Moreover, it can be considered inconvenient to propose collaborative tasks to certain types of students. The presence or absence of collaborative tasks, as well as the time at which they are presented, is described by means of course structure rules, in which collaborative tasks are included in the same way as the non-collaborative ones [5].

Group Formation. The main criterion used in this direction is the automatic grouping of students who have reached certain milestones while taking the course. The groups are formed, at a first stage, by considering the user's personal features and preferences (including aspects such as the learning style and background, among others), and their interaction style (frequency of interactions, bandwidth, etc.). Secondly, the knowledge acquired (topics visited, results while performing exercises, etc.) is considered. For each collaborative task, as soon as it is available to more than one user belonging to the same group, subgroups start to be formed, so that users can start performing the task. During the student sub-grouping, their opinions and 
preferences based on previous collaboration experiences are also considered (for example, other users they do not wish to interact with again, etc.). Finally, other default rules can also be specified (i.e., the maximum size of a group is three persons).

Problem Statement. In this work, a collaborative task is defined as "a task to check the comprehension of a particular topic, to put into practice the knowledge acquired related to a particular subject or to develop certain skills, that is performed collaboratively". The specific problem itself can be different for distinct students, although related to the same subject. When a pool of statements is provided for a particular collaborative task, the most suitable one for each group is selected depending on the problem characteristics and also on the group features and achievements while learning the involved subjects.

Collaboration Tools. Generally, students with a textual learning style prefer to propose solutions and discuss by writing texts, while those with a visual learning style usually prefer to create diagrams and draw their proposals and bases for discussion. The collaboration interface is adapted so that the most suitable tools for each group of students are presented in the main interface. The other tools will also be available. The adaptation of collaboration tools and problem statements is expressed by means of collaborative-workspace rules and collaborative-tool rules. The activation of these rules gives rise to specific collaborative workspaces, with a particular problem to be solved and a set of tools to support the cooperation [5].

Type of Collaboration. Since distance-learning systems allow the users to interact with them at any time, it is probable for the users to mainly collaborate in an asynchronous way. This communication can become synchronous when the users involved are online. The students are continuously informed which users from their group, if any, are connected, and the access to synchronous tools, if present, will be enabled so that they can go on solving their collaborative task synchronously.

Additional Support during Collaborative Task Execution. When a high degree of interaction has existed among the users in a group and yet no solution has been obtained, clues about the subjects that would be convenient for them to review may be given. It is also possible to inform them about other groups that have solved the problem (when agreed upon by these groups) for consultation purposes.

In order to reach these goals, adaptive collaborative courses are described by:

- A set of tasks to be performed, representing theoretical explanations to be studied, examples to be observed, or exercises to be solved individually or collaboratively.

- Relationships among these tasks, including decomposition, sequencing (if any) and possible dependances.

- Collaboration tools to be used for general purpose (email, forum, chat).

- Descriptions of collaborative workspaces, including the statements of the problems to be proposed and the sets of tools that will be available for each type of student.

- Adaptation rules, which determine the way in which the previous components are adapted to each student.

- Rules for student grouping. 
The course designer must provide the multimedia contents that will be used for page generation (texts, images, etc.). In order to facilitate the description of adaptive collaborative courses, an authoring tool will be developed, starting from the one used for the development of Web-based adaptive courses in TANGOW [6]. In this tool, collaborative graphical editors, forums, chats, file sharing and e-mail programs will be available, so that the course designers can select the ones that will be accessible in each case. It would be possible to include specific-purpose collaborative editors, if necessary. Concerning the student features to be considered for adaptation, the course designers will be able to select among those proposed in the authoring tool (user experience, age, learning style, etc.). They will also be able to establish other features considered as relevant for the course adaptation. All the user features and actions while interacting with the course are stored to be used with adaptation purposes.

\section{Conclusions and Current Work}

In this paper we have focused on the way adaptation and collaboration methods and techniques have been seamlessly integrated for the dynamic generation of adaptive collaborative Web-based courses. This approach is being implemented starting from the previous work done in the TANGOW system. The formalism for describing courses has been extended [5]. New modules are being developed to manage the collaboration aspects and will be integrated in the system soon. Existing adaptive courses will be turned into adaptive collaborative ones, so that this approach can be evaluated.

\section{References}

1. Brusilovsky, P.: Adaptive hypermedia. In: Kobsa, A. (ed.): User Modeling and UserAdapted Interaction, 11. Kluwer Academic Publishers (2001) 87-110

2. Barros, B., Verdejo, M.F.: Designing Workspaces to support collaborative learning. In: Pobil, A.P., Mira, J., Moonis, A. (eds.): Tasks and Methods in Applied Artificial Intelligence. LNAI, Vol. 1416. Springer-Verlag, Berlin (1998) 668-677

3. Dillembourg, P.: Collaborative learning: cognitive an computational approaches. Elsevier, Oxford (1999)

4. Schlichter, J.: Lecture 2000: More than a course across wires. Teleconference - The Business Communications Magazine. (1997) 18-21

5. Carro, R.M., Ortigosa, A., Schlichter, J.: A Rule-based Formalism for Describing Collaborative Adaptive Courses. In: Palade, V., Howlett, R., Jai, L. (eds.): KES2003. LNAI. Springer-Verlag (2003). In press.

6. Carro, R.M., Pulido, E., Rodríguez, P.: Developing and Accessing Adaptive Internetbased Courses. In: Jain, L.C., Howlett, R.J., Ichalkaranje, N.S., Tonfoni, G. (eds.): Virtual Environments for Teaching and Learning. World Scientific Publishing Company (2002) $111-149$ 